\title{
Le pathos négatif en tant que trait du discours politique totalitaire
}

The Negative Pathos in the Political Totalitarian Discourse

\section{Alicja Kacprzak}

\section{OpenEdition}

\section{Journals}

Édition électronique

URL : http://journals.openedition.org/aad/1427

DOI : 10.4000/aad.1427

ISSN : 1565-8961

Éditeur

Université de Tel-Aviv

Référence électronique

Alicja Kacprzak, "Le pathos négatif en tant que trait du discours politique totalitaire », Argumentation et Analyse du Discours [En ligne], 10 | 2013, mis en ligne le 10 avril 2013, consulté le 23 septembre 2019. URL : http://journals.openedition.org/aad/1427 ; DOI : 10.4000/aad.1427

Ce document a été généré automatiquement le 23 septembre 2019.

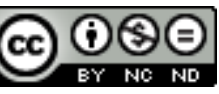

Argumentation \& analyse du discours est mis à disposition selon les termes de la licence Creative Commons Attribution - Pas d'Utilisation Commerciale - Pas de Modification 4.0 International. 


\title{
Le pathos négatif en tant que trait $\mathrm{du}$ discours politique totalitaire
}

The Negative Pathos in the Political Totalitarian Discourse

\author{
Alicja Kacprzak
}

\section{Introduction}

Chaque mouvement politique, quelle que soit son orientation, dans sa quête du pouvoir ou dans le désir de le conserver, recourt à différentes stratégies, matérielles ou symboliques, qui trouvent le plus souvent aussi leur expression langagière. Celle-ci constitue un témoignage irréfutable de l'esprit de la classe politique dans sa globalité, mais aussi de chacun des groupes en particulier. Il est évident que les idées, les convictions et les attitudes ne sont pas identiques selon les cas, ce qui explique le fait que les discours politiques, malgré leur but commun de provoquer une adhésion maximale à leurs idéologies, soient différents. Comme le constate Patrick Charaudeau (2008: 57) : «Le recours aux effets pathémiques est constitutif du discours politique», ce qui indique l'une des caractéristiques centrales de ce type de discours. Même s'il est indéniable que le discours ordinaire est lui aussi souvent marqué par l'émotion, la différence semble concerner d'une part l'intensité, et de l'autre, la fréquence relative d'affects exprimés par le discours à vocation politique. En effet, la politique constitue un espace où, plus qu'ailleurs, entre en jeu l'esprit de confrontation, entrânant inévitablement des émotions fortes, dont le propre est en plus d'être exprimées. Cependant, si l'argumentation en politique cherche d'habitude à émouvoir, le choix peut être fait quand-même entre les émotions positives et négatives à susciter auprès du public. Il semble licite de prévoir que les systèmes basés sur les valeurs de la démocratie recourent plutôt à un langage de conciliation, censé réunir les citoyens autour d'objectifs partagés. Si l'argumentation démocratique aboutit à l'émotivité, celle-ci présente plutôt un caractère affirmatif et constructif. Il n'en est pas de même pour les systèmes autoritaires, et plus particulièrement totalitaires, qui, 
indépendamment de leurs origines, semblent développer un tout autre discours, fondé sur la confrontation, le refus de l'autre, voire la haine.

Le langage totalitaire a déjà été l'objet de nombreux ouvrages qui en ont présenté des descriptions plus ou moins intégrales, parmi lesquelles se doit d'être cité l'incontournable LTI, la langue du Troisième Reich. Carnets d'un philologue de Victor Klemperer (1947), sans oublier non plus les Langages totalitaires de Jean-Pierre Faye (2004). Notons aussi une fine analyse de la "sémantique stalinienne " proposée par Aleksander Wat, écrivain polonais d'origine juive, communiste convaincu, arrêté et détenu en U.R.S.S. de 1940 à 1946, dans son ouvrage Mon siècle. Confession d'un intellectuel européen. Entretiens avec Czeslaw Milosz (1989). Parmi les travaux récents, mentionnons enfin l'apport incontestable du colloque Le langage totalitaire d'hier à aujourd'hui, en hommage à Victor Klemperer organisé en août 2010 à Cerisy, dont les contributions ont été réunies et publiées dans le volume de Victor Klemperer, repenser le langage totalitaire (2012). Les travaux énumérés, pour complets qu'ils soient, n'ont cependant pas abordé l'un des traits majeurs du discours totalitaire, à savoir sa prédilection pour l'émotivité à caractère destructeur, le pathos négatif qu'il cherche à susciter auprès de l'auditoire.

Cet article a pour but de contribuer à combler cette lacune, en montrant comment la propagande totalitaire communiste dans le bloc des pays de la soi-disant « démocratie populaire » utilisait un langage basé sur la haine et le mépris afin d'anéantir ses adversaires, c'est-à-dire tous ceux dont le seul défaut était de ne pas partager l'idéologie obligatoire. Organisant d'abord des émotions pour faire haïr l'adversaire, le pathos négatif devait servir de base émotionnelle aux actes que le régime voulait imposer à la société. Le corpus de notre analyse se compose d'une dizaine de textes authentiques, discours officiels prononcés essentiellement par les dirigeants des pays communistes, Nikita Sergueïevitch Khrouchtchev, Władysław Gomułka, Kim Il Sung, Fidel Castro, à différentes occasions dans les années 1940-1990. Ce choix historiquement et géographiquement différencié permettra de mettre en valeur l'homogénéité du discours et de ses instruments langagiers, typiques des régimes totalitaires communistes, indépendamment de leur situation chronologique et spatiale.

\section{Totalitarisme(s) et démarches totalitaires}

Cependant, avant de procéder à la présentation de ce discours particulier, il convient de revenir sur la notion même de totalitarisme, phénomène dont l'étendue dans le temps et dans l'espace suscite des discussions. En effet, depuis son apparition dans les années vingt $\mathrm{du} 20^{\mathrm{e}}$ siècle, le concept a trouvé de nombreuses définitions, prenant toutes comme point de départ le sens du mot "total», mais divergeant en ce qui concerne sa nature. Si donc la paraphrase du TLF décrivant le totalitarisme comme un "système politico-économique cherchant à imposer son mode de pensée considéré comme le seul possible» paraît suffisamment neutre pour être admise de tout le monde, la polémique s'instaure par contre quant à l'application de l'épithète « totalitaire » à tel ou tel régime.

L'un des premiers à évoquer ce terme, Paul Marion ${ }^{1}$, communiste français qui, après avoir connu la Russie soviétique, décide de quitter le Parti Communiste Français déjà en 1929 , mentionne dix ans plus tard la notion de «propagande totalitaire », cet « appel à l'homme tout entier» (1939: 183), qui «se distingue de tout autre propagande par sa persistance et son intensité» $(1939$ : 131). Le titre de son ouvrage, Leur combat : Lénine - 
Mussolini - Hitler - Franco, ne laisse pas de doute sur sa façon de concevoir l'étendue des régimes totalitaires de l'époque.

Hannah Arendt, par contre, ne voit de véritable totalitarisme qu'en Allemagne nazie et en URSS à l'époque de Staline. Si, dans son livre The Origins of Totalitarianism publié en 1951, elle note quand même des épisodes ou des tendances totalitaires même aux USA (au temps du maccarthisme), quinze ans plus tard, dans la préface de la $3^{\mathrm{e}}$ édition (1966), la philosophe allemande reprécise que les régimes poststaliniens ne sont pas totalitaires, car ils ne recourent plus à la terreur dans leur politique intérieure.

L'argument de la terreur est important aussi pour Carl Joachim Friedrich et Zbigniew Brzeziński, qui donnent pourtant une définition plus complexe du totalitarisme dans leur ouvrage publié en 1956 sous le titre de Totalitarian Dictatorship and Autocracy. Le système en question y est caractérisé comme celui qui ne tolère qu'un parti unique monopolisant le pouvoir de l'Etat, qui n'admet pas la pluralité d'information, qui se considère comme le seul à tendre vers l'accomplissement de l'humanité et qui se base sur la terreur.

Une autre conception du totalitarisme est enfin élaborée par Claude Lefort dans un cycle d'articles publiés entre 1957 et 1980 et repris ensuite en 1981 sous le titre commun L'invention démocratique. Les limites de la domination totalitaire. Selon ce philosophe, le système totalitaire se caractérise par une double "clôture " que réalisent, entre autres, les régimes communistes d'Europe de l'Est, d'Asie et d'Amérique latine. D'après lui, en premier lieu il s'agit de la destruction de l'espace public, absorbé et anéanti par le pouvoir politique : les relations sociales sont ainsi remplacées par une hiérarchie unidimensionnelle entre les dominants (le parti) et les dominés (le reste de la société). En deuxième lieu, le totalitarisme se définit par l'abolition de la pluralité des opinions, des pratiques et des objectifs, car ne comptent que l'idéologie et les cibles du régime. Ainsi, Lefort construit sa définition du totalitarisme autour de la tendance à régir la vie sociale dans toutes ses manifestations, ceci après avoir détruit l'espace public existant au préalable.

Jacques Dewitte, dans Le pouvoir de la langue et la liberté de l'esprit. Essai sur la résistance au langage totalitaire, souligne l'évolution de l'acception du terme totalitarisme, qui à l'époque de la guerre froide était utilisé pour caractériser à la fois les régimes nazi et stalinien. La façon d'appréhender ce concept a ensuite changé vers la fin des années 60 et au cours des années 70, où le mot a été « en partie discrédité [...] parce que trop lié à une intention anticommuniste ». De nos jours, poursuit Dewitte, la notion marque « un regain d'attention ", non sans rester toujours aussi controversée (2007:27).

Il est sûr qu'entre l'anéantissement moral et l'extermination physique, les camps de concentration nazis, le goulag stalinien et les prisons des dites "démocraties populaires ", les conséquences des politiques totalitaires ne sont pas les mêmes. Peutêtre, selon ce point de vue, serait-il plus juste de parler des totalitarismes et non pas du totalitarisme? Insistant sur une profonde rupture que le totalitarisme instaure entre l'État et l'Autre, c'est-à-dire la Société, Dewitte évoque aussi une sorte d'imperfection de ce type de pouvoir qui, voulant confisquer tout ce qui ne relève pas de lui, n'aboutit jamais à cette fin de manière absolue :

La société civile est l'Autre de l'État [...]. Le concept de totalitarisme désigne un pouvoir «total ", une emprise sur la réalité qui ne connaîtrait plus aucun reste et doit, pour cette raison, s'emparer de toutes les sphères de la vie sociale, comme de la vie privée et intérieure. En même temps, cette emprise ne peut jamais être totale. [...] il n'existe empiriquement aucun cas de disparition absolue de la société civile. 
C'est pourquoi on doit définir le totalitarisme comme une visée, un projet, une intention ou une ambition (Dewitte 2007 :27-28).

Cependant, même si, selon les cas, les démarches totalitaires de l'exclusion de l'adversaire utilisent des instruments différents et aboutissent à des effets variables, leur nature reste identique: c'est l'existence de l'Autre qui de façon apparemment contradictoire nourrit l'unité du système totalitaire (Lefort 1981 : 176), car l'Autre n'est jamais innocent, son altérité fait de lui un ennemi qu'il faut détecter, démasquer et éliminer. Cette forclusion de l'Autre qu'évoque aussi Dewitte constitue à notre avis le noyau même de la définition du totalitarisme. La formule semble d'ailleurs d'autant plus valable qu'aux dires du philosophe, au-delà du champ politique et social, elle n'exclut pas d'autres domaines d'expérience, notamment la littérature et l'esthétique (Dewitte 2007 : 26).

\section{L'Autre et la « construction de l'ennemi »}

Puisque les totalitarismes se fondent paradoxalement sur l'existence de l'Autre, la poursuite de l'adversaire ne se termine jamais, si bien que la face de l'adversaire doit être constamment renouvelée. Comme le dit Lefort (1981: 173), «la constitution du peuple-Un exige la production incessante d'ennemis », leur " invention ». En effet, leur éventail semble infini, à commencer, en ce qui concerne les régimes staliniens et poststaliniens, par l' «ennemi du peuple» ${ }^{2}$, terme connu pourtant depuis la Rome antique et repris, entre autres, dans le décret du 11 décembre $1917^{3}$ signé par Lénine, Trotski et Staline. La propagande communiste exploitera aussi à fond ses synonymes (dont on évoque un échantillon représentatif, recueilli dans le corpus de textes indiqués dans la bibliographie du présent article), suffisamment vagues pour pouvoir être appliqués à tout un chacun, selon les besoins du régime: «ennemi du communisme », « ennemi de la République Populaire ", " ennemi des travailleurs ", " ennemi du prolétariat», "adversaire du socialisme ", "élément antisocialiste ", « individu idéologiquement étranger », « ennemi de classe ». Fidel Castro verra, quant à lui, la nécessité de préciser cette dernière catégorie en la divisant en deux groupes, en affirmant en juillet 1973 : «Dans la lutte, il [le peuple] a appris à connaître ses ennemis de classe internes et externes ${ }^{4}$. Cette distinction est d'ailleurs bien connue et reconnue dans la rhétorique communiste générale : quelle que soit la langue nationale utilisée, le premier groupe est constitué par ceux qui, comme «banquiers", «fabricants ", " riches paysans ", mais aussi «feignants » et "saboteurs ", selon une formule anonyme consacrée, « parasitent la chair saine du peuple travailleur des villes et des villages». Cette catégorie comporte aussi ceux qui, selon une autre formule consacrée, "ont trahi la cause », à savoir : les "traîtres aux intérêts de la révolution », les «vilains traîtres de la patrie», les "réactionnaires», les «vendus », les «mercenaires du tsarisme», les «hobereaux de la bourgeoisie», les «salariés du capitalisme », les « laquais des Américains ». Le second groupe des ennemis de classe se compose par contre de ceux qui menacent le système de l'extérieur: "espions ", "vermine bourgeoise de l'étranger», "gouvernements étrangers tyranniques et oppresseurs", sans oublier un nom collectif disqualifiant, aussi vulgaire que fréquemment employé : "l'Ouest pourri ». Ces catégories stéréotypées ont d'ailleurs souvent trouvé dans la propagande communiste une expression aussi réaliste 
qu'exagérée au travers de différents supports artistiques, pièces de théâtre, films, peinture ou affiches ${ }^{5}$.

Il est intéressant de constater que le répertoire de dénominations citées, selon un point de vue fonctionnel, pourrait être sans doute divisé en deux catégories. En effet, certaines appellations semblent avoir un caractère universel (dans le cadre des dites "démocraties populaires»), à commencer par l'éternel "ennemi », quel que soit le complément déterminatif qui accompagne ce terme. D'autres, par contre, présentent un caractère plus spécifique et de ce fait ne sont actualisées que par des circonstances particulières d'un pays et / ou d'une époque, tels "mercenaires du tsarisme » ou "laquais des Américains". Ce thème mériterait sans doute une analyse et un développement approfondis qui, faute de place, ne peuvent être faits ici.

La poursuite de l'ennemi en tant qu'élément constitutif du totalitarisme ne saurait rester sans incidence sur le langage utilisé par le régime. En effet, la topique de l'ennemi fait office de source renouvelable d'émotions négatives que le discours politique totalitaire cherche à transférer à l'auditoire. Celui-ci ne se laisse pas facilement déterminer: s'agit-il seulement de ceux qui, rassemblés dans des réunions plus ou moins obligatoires selon le régime, sont soumis à un rituel semblable à celui de l'orwellienne " heure de la haine », ou bien aussi de ceux qui, à force de vivre dans une société opprimée par le totalitarisme, ne peuvent pas, malgré eux, échapper à sa propagande omniprésente? C'est ce dernier élément qui doit être pris en considération, lorsqu'on évoque l'auditoire d'une adresse totalitaire. Pour d'autres motifs sans doute, La nouvelle rhétorique définit d'ailleurs aussi l'auditoire comme l'ensemble de tous ceux que l'orateur veut persuader :

On voit immédiatement [...] combien il est difficile de déterminer, à l'aide de critères purement matériels, l'auditoire de celui qui parle [...]. C'est la raison pour laquelle, il nous semble préférable de définir l'auditoire, en matière rhétorique, comme l'ensemble de ceux sur lesquels l'orateur veut influer par son argumentation (Perelman \& Obrechts-Tyteca $1958: 25$ ).

Même si l'on admet que les émotions sont complètement dissociées de la raison, il semble qu'elles soient inséparables d'un jugement préalable qui permet d'accepter ou de rejeter l'objet sur lequel elles portent. La recherche de l'adversaire trouve ainsi son expression dans le discours totalitaire par la technique de la «construction de l'ennemi ", consistant à juger et à présenter l'adversaire, quelle que soit sa nature, comme responsable de quelque chose, ce à quoi le Système se voit obligé de réagir. La réaction qui vise l'adversaire s'adresse cependant à l'auditoire censé partager les émotions violentes que celui-là inspire, telles que le mépris, l'indignation et la haine.

Parmi les instruments langagiers fondateurs de cette technique, citons avant tout des figures d'opposition servant à déterminer l'Autre et les caractéristiques de celui-ci sur lesquelles portera ensuite l'argumentation. Le discours prononcé par Władysław Gomułka, premier secrétaire du Parti Ouvrier Polonais (PPR $)^{6}$, lors de la première assemblée de ce groupement le 6 décembre 1945 offre un bon exemple de ce procédé. Fondé en 1942 par les communistes polonais à l'initiative de Staline, le PPR devait remplacer le Parti Communiste Polonais qui, considéré par Staline comme "trotskiste», avait perdu la totalité de ses membres lors des Grandes Purges soviétiques des années 1930. Gomułka, qui doit expliquer l'apparition du nouveau parti sur les cendres de l'ancien, recourt au moyen bien connu de la propagande communiste, celui consistant à avilir le parti précédent devenu inexistant, en le plaçant 
au même niveau que tout « ce qui en ancienne Pologne était pourri et mauvais, nuisible et dangereux » :

Toutes les erreurs du passé du mouvement ouvrier en Pologne ne lui permettaient pas de prendre sa place auprès du peuple polonais [...]. Nous sommes un parti nouveau de ce mouvement, un parti nouveau du peuple travailleur des villes et des villages [...]. Nous sommes un parti jeune, car nous avons rejeté tout ce qui dans le passé a nui aux intérêts de la classe ouvrière et des masses travailleuses, tout ce qui n'a pas permis aux anciens partis de mener ces masses sur le chemin de l'indépendance sociale, sur le chemin de la construction de la démocratie populaire [...]. Notre jeunesse exempte des fautes de la vieillesse d'autres partis nous permet aujourd'hui de bâtir de la manière la plus conséquente la démocratie polonaise et les fondements inébranlables de l'indépendance du pays. C'est pour cela que notre parti non seulement n'a pas honte de sa jeunesse, mais encore peut en être fier [...]. Dans les rangs de notre parti, il n'y a pas de place pour les gens de la vie commode et facile et du travail léger. Les gens comme cela ne pourraient pas construire la nouvelle Pologne et la nouvelle vie. [...] Nous voulons liquider absolument tout ce qui en ancienne Pologne était pourri et mauvais, nuisible et dangereux. Nous voulons abolir tous les obstacles qui se dressent sur notre chemin, et nous les abolirons. Car nous sommes un parti de lutte (Gomułka 1945 : 34).

Le recours constant à l'antithèse fondée sur l'antonymie ancien / nouveau, vieillesse / jeunesse sert de cadre à la présentation des deux partis. Des contextes négatifs pour le passé se répètent («erreurs du passé » " fautes de la vieillesse », « tout ce qui dans le passé a nui aux intérêts de la classe ouvrière et des masses travailleuses ») et restent en opposition par rapport à la topique de l'avenir prometteur, marquée par des formulations à visée positive (" un parti nouveau du peuple travailleur", "un parti jeune»), si bien que la figure est réitérée dans le discours et que la distribution entre le pôle du passé et de l'avenir s'accentue. La triple répétition du substantif «chemin » semble, quant à elle, aussi significative, renvoyant avec force à un but à poursuivre ; avec les verbes «bâtir » et "construire » symbolisant l'ordre nouveau ils contrastent avec le syntagme « tous les obstacles qui se dressent sur notre chemin » qui fait allusion à l'ordre ancien. C'est dans ce jeu d'oppositions que l'ennemi est déterminé : il s'agit d'« anciens partis », d'« autres partis », que Gomułka ne nomme d'ailleurs pas, mais qui en tant que représentant « les gens de la vie commode et facile et du travail léger »sont adversaires de « la classe ouvrière et des masses travailleuses ».

À la marge, remarquons que, par son message, l'orateur cherche en même temps à fonder l'ethos du nouveau parti. En recourant constamment à l'emploi de la première personne du pluriel, le pronom « nous » et le possessif « notre », il dresse l'image moins d'une organisation hiérarchique que d'une communauté agissant en bloc, réunie « autour d'une pensée unique et sans fissure » (Amossy 2010 : 166). Il est caractéristique aussi que, dans la Pologne à l'issue de la Seconde Guerre mondiale, Gomułka s'appuie non seulement sur les valeurs de "l'indépendance sociale» et de "la démocratie populaire ", propres aux mouvements ouvriers, mais qu'il évoque en plus celle des "fondements inébranlables de l'indépendance du pays ", ceci sans doute pour apaiser l'opinion publique effarée déjà en hiver 1945 par l'ordre politique soviétique en train de s'installer en Pologne.

La topique de l'ennemi étant essentielle dans le langage totalitaire, il n'est pas étonnant qu'elle soit présente aussi bien dans le discours se rapportant à la politique intérieure des pays communistes, qu'à leur politique extérieure. Le passage ci-dessous concernant la politique internationale de l'URSS est tiré du discours de Nikita Khrouchtchev prononcé lors du meeting pour l'amitié entre les peuples de l'Union Soviétique et de la 
République de Cuba le 23 mai 1963 à Moscou. Le dirigeant soviétique commente à sa façon la situation qui, après la crise de Cuba en octobre 1962, a placé le monde au bord de la guerre nucléaire, ayant opposé les États-Unis et l'Union Soviétique au sujet des fusées nucléaires soviétiques pointées vers le territoire des États-Unis depuis l'île de Cuba :

Le comportement impérialiste conduit par les États-Unis d'Amérique a beau vouloir arrêter ou freiner le grand processus révolutionnaire de la libération de l'humanité, elle n'en a pas la force. Les peuples qui luttent pour la liberté et l'indépendance sont capables avec l'appui de toutes les forces de la paix et du socialisme, de défendre leurs conquêtes [...]. Maintenant, six mois après ces événements, toute la gravité du danger qui pesait sur le monde par suite des agissements perfides des forces agressives de l'impérialisme américain, ressort plus clairement encore [...]. Les calculs des impérialistes qui voulaient étouffer la révolution cubaine, furent déjoués grâce à la ferme attitude du gouvernement de la République cubaine présidé par le camarade Fidel Castro, grâce à la cohésion combative du peuple cubain, grâce à l'aide militaire de l'Union Soviétique ainsi qu'au puissant soutien moral et politique des pays socialistes, de tous les peuples épris de paix qui se sont dressés en un front uni pour défendre l'héroïque île de la Liberté (Khrouchtchev 1963) ${ }^{7}$.

Khrouchtchev désigne les États-Unis d'Amérique comme coupables de la crise par une argumentation basée aussi sur une antithèse, qui met fortement en contraste «le comportement impérialiste conduit par les États-Unis d'Amérique» et «le grand processus révolutionnaire de la libération de l'humanité». Cette opposition se trouve renforcée par plusieurs réformulations évoquant vigoureusement d'un côté « la gravité du danger ", "les calculs des impérialistes", "des agissements perfides des forces agressives de l'impérialisme américain », et de l'autre "les peuples qui luttent pour la liberté et l'indépendance » et «tous les peuples épris de paix». L'emploi anaphorique de la préposition "grâce à » amplifie le sentiment de gratitude envers les instances censées déjouer les plans de l'adversaire, à savoir le gouvernement et le peuple de la République cubaine, soutien militaire et moral de l'Union Soviétique, ainsi que d'autres pays socialistes. Cette répétition de "grâce à ", terme ayant par ailleurs une valeur positive, renforce au contraire dans le discours du premier secrétaire soviétique l'impression du danger miraculeusement contourné, mais toujours réel, tant qu'existe l'ennemi si clairement indiqué.

\section{L'affectivité dans le discours}

Les faits blâmables rapportés et commentés par les orateurs communistes ne devaient pas laisser l'auditoire indifférent. Or, l'efficacité de la propagande se confirme au moment où le public adhère à ses jugements et partage ses émotions. Dans le discours, l'affectivité revêt, selon Ruth Amossy (2006: 187), deux formes principales : soit les émotions provoquées auprès du public restent implicites, soit elles sont mentionnées explicitement.

\subsection{L'affectivité implicite}

L'extrait suivant du discours de Kim Il Sung, prononcé devant les délégués au Congrès pan-national le 18 août 1990, peut illustrer la première forme. Le premier secrétaire du parti nord-coréen esquisse l'image défavorable du pays ennemi, les États-Unis, pour montrer l'infériorité morale du capitalisme : 
Dans le monde capitaliste, les États-Unis sont le pays le plus développé, disons-le, mais c'est un pays corrompu et malade: les inégalités sociales y sont prononcées, dont celle des richesses, et toutes sortes de vices sociaux y sévissent. Les riches y vivent dans le luxe, tandis qu'un grand nombre de personnes y souffrent de la faim et vivent dans les rues faute de logements. C'est le pays qui dénombre le plus de cas de meurtres, de brigandage, le plus grand nombre de drogués et d'alcooliques, où le sida est le plus répandu. La démocratie à l'américaine est au service d'une minorité de privilégiés, et non au service des masses populaires. [...]. Il serait stupide de se nourrir d'illusions sur les Etats-Unis et d'essayer d'imiter la démocratie à l'américaine ${ }^{8}$.

Cette description commence à nouveau par une antithèse dessinant un contraste entre l'économie très développée des États-Unis et la bassesse morale de son système social «corrompu et malade». La figure s'appuie d'abord sur l'antonymie entre les noms "luxe» et "faim ", puis sur celle entre le substantif "riches » et le syntagme " un grand nombre de personnes [qui] y souffrent de la faim et vivent dans les rues faute de logements». Un accent supplémentaire porte sur le petit nombre de riches (signalé plus loin aussi par la formulation «minorité de privilégiés ») et sur le grand nombre de pauvres du pays (signalé aussi par le terme "masses populaires »). L'opposition entre les deux mondes n'est pas cependant équilibrée, car les évocations de l'infériorité morale du capitalisme se multiplient au point de noyer l'idée du bon niveau économique des USA. Une accumulation d'éléments désavouant la vie dans le pays où fleurissent "meurtres ", " brigandage ", "drogués ", " alcooliques ", « sida » cherche à susciter des affects négatifs auprès de l'auditoire nord-coréen. Ces sentiments ne sont pas indiqués directement dans le texte qui vise à la mobilisation affective de l'auditoire, menant celui-ci à " une conclusion émotionnelle ", pour reprendre l'expression de Ruth Amossy (2006 : 189) d'horreur et de haine.

\subsection{L'affectivité explicite directe et indirecte}

Pour ce qui est de l'expression explicite des émotions de l'auditoire, celles-ci peuvent trouver leur issue d'une manière directe ou indirecte. Dans le premier cas, il s'agit d'un procédé souvent pratiqué par des systèmes totalitaires, consistant à faire parler « des représentants du peuple» censés représenter l'auditoire. En effet, pour faire valoir auprès de la société certaines opinions, les régimes en question admettaient qu'elles soient exprimées à la tribune par des "citoyens moyens", parfois réellement convaincus de ce qu'ils devaient dire, parfois complices du régime, parfois, par contre, manipulés ou forcés. Il s'agissait dans ce cas d'une vraie mise en scène qui aboutissait à la prise de parole d'un "travailleur indigné » dont le rôle était de manifester l'avis apparemment partagé par tout le peuple. Un exemple de cette procédure est offert par le commentaire public prononcé par un travailleur lors d'un meeting organisé afin de condamner les protestations ouvrières dues à l'augmentation des prix en juin 1976 dans deux villes industrielles polonaises, Radom et Ursus : "J'ai honte pour ceux de Radom et surtout pour ceux d'Ursus. Il s'est avéré que notre société comprend encore des gens qui ne sont ni meilleurs ni pires que la noblesse bornée et rebelle des $17^{\mathrm{e}}$ et $18^{\mathrm{e}}$ siècles! » (Travailleur anonyme 1976) 9 .

L'exclamation à la première personne " j'ai honte » indique d'une manière explicite et directe le sentiment d'humiliation que l'orateur attribue au peuple entier; en effet, il doit produire " un effet de contagion", compris comme une influence du locuteur sur le récepteur (Amossy 2006 : 195). L'indignation est justifiée d'une façon supplémentaire 
par l'évocation de "la noblesse bornée et rebelle des $17^{\mathrm{e}}$ et $18^{\mathrm{e}}$ siècles», formule dénotant dans la propagande communiste les responsables de la chute de la République Polonaise à la fin du $18^{\mathrm{e}}$ siècle. Cette allusion n'est évidemment pas innocente, car il s'agit de rappeler aux Polonais que les libertés de la noblesse, dont la liberté d'expression, ont déjà une fois mené le pays au désastre. L'argumentation trouve ainsi le support de la catégorisation analogique faisant intervenir l'expérience de la communauté socio-culturelle. L'auditoire est censé être réuni par l'image puisée dans la mémoire partagée et le «je » de l'orateur n'a pas d'autre fonction que de rassembler d'avantage la collectivité dont il n'est que le porte-parole.

Les émotions de l'auditoire peuvent aussi être exprimées d'une manière explicite indirecte, à savoir par l'orateur qui décrit la réaction de l'auditoire à propos d'une situation censée générer de fortes émotions. L'exemple ci-dessous provient du discours de Władysław Gomułka, prononcé le 19 mars 1968 pendant une grande réunion d'activistes du Parti Ouvrier Unifié Polonais ${ }^{10}$. C'est le moment de l'une des plus grandes crises du régime communiste en Pologne et le meeting, censé avoir un grand impact de propagande, est transmis par la télévision polonaise, si bien que son auditoire immédiat est particulièrement nombreux. Gomułka commente, dans le style très émotif qui le caractérise, les manifestations d'étudiants polonais, brutalement réprimées par l'État:

Au cours des dix derniers jours, des événements importants ont eu lieu dans notre pays. Une grande partie de la jeunesse estudiantine [...] a été trompée et conduite par des forces ennemies au socialisme sur une fausse route [...]. Tout cela a profondément indigné la société. Et c'est surtout la classe ouvrière qui s'est vigoureusement opposée aux inspirateurs et aux organisateurs de ces actions illégales d'étudiants. Tout le pays a été submergé par une onde de réunions massives et de meetings de milliers d'ouvriers, d'habitants des villes et des villages, pendant lesquels une voix puissante a exigé d'indiquer à la société de vrais responsables de cette situation et de punir sévèrement tous les coupables (Gomułka 1969 : 78).

Excédé par les événements qui étaient en train de prendre une ampleur de plus en plus considérable dans tout le pays, Gomułka fait appel aux émotions de l'auditoire en évoquant une fois de plus " des forces ennemies au socialisme » et leur action néfaste sur «la jeunesse estudiantine ». Le dirigeant, à la tribune du peuple, exprime lui-même les sentiments qu'il impute à la totalité des Polonais: "Tout cela a profondément indigné la société ». Le verbe "indigner", traduisant un sentiment très fort, est employé certes à la troisième personne du singulier, mais ce n'est que pour illustrer l'émotion de l'auditoire que l'orateur représente par défaut. L'émotion présumée de la société entière se trouve présentée dans ce discours comme " une voix puissante » qui réclame de trouver et de punir «de vrais responsables de cette situation et tous les coupables".

\section{Les instruments lexicaux du pathos négatif}

La stratégie de la construction de l'ennemi, si systématique dans le discours totalitaire, s'accompagne nécessairement d'emplois lexicaux particuliers, destinés à disqualifier l'adversaire, à provoquer le dédain et la haine. Sur la base du corpus réuni et traité ici globalement, il est possible d'en distinguer deux groupes principaux, selon leur poids 
dans le discours: le premier comporte des mots intrinsèquement dépréciatifs et le second tient sa valeur dépréciative de son contexte ${ }^{11}$.

\subsection{Lexique à valeur essentiellement dépréciative}

A ce niveau, on relève entre autres :

a. des substantifs ou expressions constituant des appellations insultantes individuelles qui se rapportent à l'activité considérée comme nuisible et malhonnête, telles qu' " embrouilleur de l'ordre public », "traître à la patrie ", "feignant", " espion », « spéculant », « déserteur », « saboteur », etc.

b. des substantifs constituant des appellations insultantes collectives désignant des groupes considérés comme criminels, ou ayant perdu des qualités humaines, comme par exemple " bande ", « coterie ", « clique ", « ramassis ", « maffia », « meute », etc.

c. des adjectifs qui évoquent des qualités blâmables de l'ennemi politique, comme " réactionnaire ", " faux ", « mensonger ", « meurtrier ", « sale ", « pourri », etc.

d. des verbes qui dénotent des actions répréhensibles de l'adversaire, tels " instiguer », « diffamer », « exhaler du venin », « brader », « vendre », « mordre », « ronger », etc.

\subsection{Lexique dont la valeur dépréciative est actualisée par le contexte}

Comme le constate Laurence Rosier (2009) à propos de l'insulte, tout mot, compte tenu de son contexte d'utilisation, peut devenir péjoratif. En particulier, cette modification sémantico-pragmatique peut s'opérer au moyen des procédés suivants :

4.2.1. Juxtaposition d'un élément neutre avec des éléments négatifs qui lui transmettent leur coloration péjorative. Ainsi, un syntagme composé de mots n'ayant pas de nuance négative inhérente, à savoir par exemple « des gens idéologiquement étrangers ", qui, selon le contexte, pourrait être considéré comme neutre, acquiert une nuance péjorative au contact d'une énumération de termes négatifs: «des débrouillards, des carriéristes, des opportunistes non-idéalistes et des adversaires masqués» dans le fragment du discours de Władysław Gomułka prononcé le 19 mars 1968: «Notre parti renforce son autorité en se purifiant des gens idéologiquement étrangers, des débrouillards, des carriéristes, des opportunistes non-idéalistes [...] et des adversaires masqués» (Gomułka 1969 : 81).

4.2.2. Antonymie d'un mot dont la valeur négative résulte du contraste avec un terme voisin ayant une valeur positive. Dans le même discours du 19 mars 1968, Gomułka oppose ainsi le terme «fossoyeur » au terme positif « une partie consciente de la classe ouvrière »: «Ce n'est qu'une partie consciente de la classe ouvrière qui a mené une lutte conséquente contre les fossoyeurs de la Pologne à l'époque de l'entre-deuxguerres». Ce procédé rabaisse la valeur du premier mot, surtout avec la présence du complément déterminatif «de la Pologne à l'époque de l'entre-deux-guerres » qui lui ajoute une autre nuance négative.

4.2.3. Emploi d'un mot par antiphrase qui lui fait prendre en raison du contexte une valeur péjorative. Dans la phrase tirée de son discours du 19 mars 1968, Władysław Gomułka constate: "Ces donations et ces subventions proviennent du travail du peuple, du travail de ceux que sa majesté l'ennemi de la Pologne populaire, Kisielewski, a traité de crétins » (Gomułka 1969: 83). Le titre dérisoire «sa majesté », par lequel il 
accompagne le patronyme d'un opposant du régime, Kisielewski, revêt une forte nuance négative au contact, d'abord, du mot "ennemi ", mais aussi du fait de la proximité de l'appellation "Pologne populaire», rappelant qu'il s'agit d'un Etat prolétaire qui proscrit par sa nature les titres de ce type.

4.2.4. Péjoration d'un mot par des contextes négatifs fréquents. C'est notamment le cas du mot «capitaliste»dans le discours communiste en général. Utilisé par la propagande du régime, il perd son sens dénotatif de « celui qui possède les moyens de production et en contrôle l'emploi " (selon la définition du TLF), pour prendre inévitablement la valeur connotative de 'celui qui, possédant les moyens de production et en contrôlant l'emploi, exploite la classe ouvrière'. Voici un exemple de ce procédé très fréquemment employé, provenant d'un discours de Fidel Castro du 26 juin 1991, dans lequel une série de questions rhétoriques est produite pour démasquer le régime ennemi comme générateur de toutes les calamités de la vie sociale et économique, et comme touchant même à l'indépendance du pays :

Je me demandais : mais d'où vient l'injustice? d'où vient l'inégalité ? d'où vient la pauvreté? d'où vient le sous-développement? d'où viennent toutes ces calamités, sinon du capitalisme? et le néocolonialisme et l'impérialisme, sinon du capitalisme ${ }^{12}$ ? (Castro 1991)

$\mathrm{Au}$ contact des mots désignant des concepts considérés comme négatifs, "injustice ", "inégalité ", "pauvreté ", "sous-développement", " néocolonialisme ", « impérialisme », le mot « capitalisme » acquiert lui aussi une coloration péjorative.

\section{Les instruments lexico-syntaxiques du pathos négatif}

\subsection{Cascades de mots dépréciatifs}

Notons un autre procédé qui constitue une pratique bien particulière du discours totalitaire et que l'on pourrait définir comme lexico-syntaxique, car il consiste en une accumulation presque pathologique d'insultes de tout type, formant une sorte de 'cascade de mots dépréciatifs'. Le fragment du discours de Władysław Gomulka du 17 octobre 1959, consacré à la présentation de la situation difficile du marché de la viande en Pologne, en constitue un exemple significatif :

Camarades! Pendant cette réunion, nous présentons au parti, à la classe ouvrière, à la nation, d'une manière honnête et ouverte les motifs qui nous ont poussés à l'augmentation des prix de la viande, ainsi que nos projets et tâches pour créer les meilleures conditions de développement pour notre économie. Contrairement à ce qu'affirment les adversaires du socialisme, les ennemis de la Pologne populaire, les journalistes menteurs, correspondants de revues bourgeoises et les mercenaires avilis par la trahison de leur patrie, notre économie est basée sur des principes justes et se développe sans cesse (Gomułka 1959 : 47).

Une longue explication (que nous ne citons pas) des raisons de l'augmentation des prix de la viande ${ }^{13}$, illustrée par de nombreux tableaux et chiffres, cède brusquement la place à une suite d'invectives adressées aux critiques du système: "adversaires du socialisme », "ennemis de la Pologne Populaire», "journalistes menteurs", " correspondants de revues bourgeoises", «mercenaires avilis par la trahison de leur patrie ». Cette énumération de mots dépréciatifs qui descendent en cascade semble être 
une technique destinée à noyer l'auditeur sous un déluge d'hostilités auquel il ne peut que souscrire.

Le même procédé langagier est employé par Fidel Castro dans son discours prononcé le 26 juillet 1961 à La Havane :

La réaction et la contre-révolution ne lèveront pas la tête, [...] la contre-révolution ne lèvera pas la tête parce que le peuple est contre elle, parce que le peuple ne chérit pas les privilégiés, ne chérit pas les exploiteurs, ne chérit pas la vermine, ne chérit pas les parasites, ne chérit pas les traîtres, ne chérit pas les menteurs, ne chérit pas les vendeurs de la patrie, ne chérit pas les lèches-bottes de l'impérialisme, ne chérit pas les ennemis des travailleurs, les ennemis des paysans, les ennemis des étudiants, les ennemis de notre jeunesse, les ennemis de notre société, les ennemis de notre nation, les ennemis de notre avenir, les ennemis de notre progrès (Castro 1961) ${ }^{14}$.

L'impétuosité des vitupérants, tels "privilégiés », " exploiteurs », "vermine », "parasites », «traitres ", " menteurs ", "vendeurs de la patrie », « lèches-bottes de l'impérialisme ", "ennemis des travailleurs ", dont Castro accable les adversaires politiques, est augmentée par l'octuple répétition du syntagme " ne chérit pas ». Cette anaphore rhétorique cède presqu'immédiatement la place à une autre, dans laquelle c'est le terme " ennemis » qui devient récurrent, lui aussi répété huit fois. Le discours du dirigeant cubain acquiert de ce fait une sorte de symétrie et un rythme puissant qui communiquent plus d'énergie destructrice à ses invectives.

\subsection{Séries de mots grossiers et choquants}

Notons aussi la prédilection portée par le discours totalitaire à des mots grossiers, choquants, exagérés ("vermine ", "parasites ») qui, en apparaissant dans des séries, augmentent encore leur valeur négative. Un exemple évocateur de cette technique est constitué par l'extrait d'un discours de Nikita Sergueïevitch Khrouchtchev qui, s'adressant à des écrivains soviétiques critiquant le régime, s'écrie : « Vous vous faites l'auteur d'un livre à sensation, vous servez ce plat et qui le consomme? Ce plat séduit, comme la charogne, les mouches, les grosses et sales mouches et toute la vermine bourgeoise de l'étranger»(Khrouchtchev1963:35) ${ }^{15}$.

Sans condamner directement les auteurs de « livres à sensation ", auxquels le dirigeant du parti soviétique semble donner le conseil presque débonnaire de ne pas dépasser les limites de la critique autorisée, Khrouchtchev vise par son attaque fulgurante le monde capitaliste de l'Occident. Les épithètes qui se rapportent à celui-ci prennent la forme d'une gradation dans laquelle des termes d'intensité négative croissante se succèdent: « les mouches, les grosses et sales mouches ", tout en renvoyant à « la charogne » qui constituerait leur proie. Le dernier terme de la gradation, «toute la vermine bourgeoise de l'étranger ", apporte l'effet négatif le plus marquant de l'énumération, car il résume la haine et le mépris à l'encontre de l'ennemi ainsi désigné. Basée sur le choix de termes injurieux particulièrement disqualifiants, l'emphase de la gradation instaure une communication pathémique qui fait partager à l'auditoire l'émotion de l'orateur. 


\section{Conclusion}

Le discours totalitaire qui peut être compris comme une sorte de discours de domination, indépendamment de ses origines, tend à se superposer à tout dialogue social, en excluant des valeurs autres que celles qu'il prône lui-même. En accord avec le principe que "toute argumentation est l'indice d'un doute " (Perelman et ObrechtsTyteca $1988: 635$ ), ce type de discours ne cherche pas à cumuler les arguments, mais bien au contraire, il présente des opinions comme des faits, en laissant croire qu'il ne doit pas les justifier, tellement ils seraient évidents. Cette absence d'arguments bien fondés, si typique des discours totalitaires, prend les allures d'une argumentation contraignante qui rend superflue toute preuve ultérieure. Analysé à la lumière des discours officiels des dirigeants de quelques pays dits "démocraties populaires", Union Soviétique, Pologne, Corée du Nord et Cuba des années 1940 - 1990, ce genre discursif apparaît comme thématiquement et stylistiquement homogène, dont une caractéristique essentielle reste sans doute son contenu émotionnel particulier. S'il est certain qu'il comporte aussi des éléments d'éloge pour glorifier le régime, donc des éléments visant à déclencher une affectivité positive, la proportion de ceux-ci semble minoritaire par rapport à l'industrie de la haine déployée par sa propagande, en accord avec le principe évoqué, entre autres, par Christine Tappolet (2000), que certaines valeurs appellent, plus que d'autres, un certain type d'émotions. Ainsi, les idéologies totalitaires, fondées sur le conflit, ont inévitablement recours au pathos négatif qui se place de cette manière, avec son éventail de procédés langagiers particuliers, parmi les traits génériques qui les définissent.

\section{BIBLIOGRAPHIE}

Achard, Guy, 1981. Pratique rhétorique et idéologie politique dans les discours « Optimates » de Cicéron

(Leiden : E. J. Brill)

Amossy, Ruth. 1997. « Cliché et pathos : l'instigation à la violence ", Raynaud, Claudine \& Peter Vernon (éds), Fonctions du cliché. Du banal à la violence, Graat 16 (Tours), 15-28

Amossy, Ruth. 2006 [2000]. L'argumentation dans le discours (Paris : Armand Colin)

Amossy, Ruth. 2010. La présentation de soi. Ethos et identité verbale (Paris : PUF)

Arendt, Hannah. 1966 [1951]. The Origins of Totalitarianism, 3 vol. (New York : Harcourt Brace \& Co)

Bralczyk, Jerzy. 2007. O jĘzyku propagandy i polityki (Warszawa : Trio)

Charaudeau, Patrick. 2008. "Pathos et discours politique », Rinn, Michael (éd.). Émotions et discours. L'usage des passions dans la langue (Rennes : PUR), 49-58

Charaudeau, Patrick \& Dominique Maingueneau. 2002. Dictionnaire d'analyse du discours (Paris : Seuil) 
Dewitte, Jacques. 2007. Le pouvoir de la langue et la liberté de l'esprit. Essai sur la résistance au langage totalitaire (Paris : Éditions Michalon)

Faye, Jean-Pierre. 2004 [1972]. Langages totalitaires (Paris : Hermann)

Friedrich, Carl Joachim \& Zbigniew Brzezinski. 1956. Totalitarian Dictatorship and Autocracy (New York : Harper Row)

Kacprzak, Alicja. 2012. « La propagande totalitaire et ses instruments discursifs (le cas de la calomnie et de l'invective) ", Aubry, Laurence \& Béatrice Turpin (éds). Victor Klemperer. Repenserle langage totalitaire aujourd'hui (Paris : CNRS Éditions), 119-131

Kacprzak, Alicja. 2009. « Comment ridiculiser l'ennemi politique », Marillaud, Pierre \& Robert Gauthier (éds). Humour, ironie et les discours (Toulouse : Univ. Toulouse - Le Mirail), 357-368

Kamińska-Szmaj, Irena. 2004. « Propaganda, perswazja, manipulacja - próba uporzĄdkowania pojĘć », Krzyżanowski, Piotr \& Paweł Nowak (éds). Manipulacja wjĘzyku, (Lublin : Wydawnictwo Uniwersytetu Marie Curie-Skłodowskiej), 13-27

Kamińska-Szmaj, Irena. 2007. Agresja jĘzykowa w życiu publicznym. Leksykon inwektyw politycznych 1918-2000 (Wrocław : Wydawnictwo Uniwersytetu Wrocławskiego)

Klemperer, Victor. 1996 [1947]. LTI, la langue du Troisième Reich. Carnets d'un philologue (Paris : Albin Michel)

Koren, Roselyne. 1991. «Violence verbale et argumentation dans la presse révolutionnaire et contre-révolutionnaire ", Presse d'élite, presse populaire et propagande pendant la Révolution française. Studies on Voltaire and the Eighteenth Century 287 (Oxford : The Voltaire Foundation), 319-334 Lefort, Claude. 1981. L'invention démocratique. Les limites de la domination totalitaire (Paris : Fayard) Marion, Paul. 1939. Leur combat : Lénine - Mussolini - Hitler - Franco (Paris : Librairie Arthème Fayard)

Perelman, Chaïm \& Lucie Obrechts-Tyteca. 1988 [1958]. La nouvelle rhétorique, Traité de l'argumentation (Bruxelles : Éditions de l'Université de Bruxelles)

Plantin, Christian. 2005. L'Argumentation (Paris : PUF)

Rinn, Michael (éd.). 2008. Émotions et discours. L'usage des passions dans la langue (Rennes : PUR)

Robrieux, Jean-Jacques. 2000. Rhétorique et argumentation (Paris : Nathan)

Rosier, Laurence. 2009 [2006]. Petit traité de l'insulte (Bruxelles : Espaces de Libertés, Edition du centre d'action laïque)

Tappolet, Christine. 2000. Émotions et valeurs (Paris : PUF)

Vincent, Diane \& Geneviève Bernard Barbeau. 2012. «Insulte, disqualification, persuasion et tropes communicationnels : à qui l'insulte profite-t-elle? ", Argumentation et Analyse du Discours [En ligne], 8|2012, Consulté le 23 juillet 2012. URL : http://aad.revues.org/1252

Wat, Aleksander. 1989. Mon siècle. Confession d'un intellectuel européen. Entretiens avec Czeslaw Milosz (Paris - Lausanne : De Fallois/ L'Âge d'Homme)

\section{Corpus}

Gomułka, Władysław. 1959. Aktualne trudności na rynku miĘsnym i środki niezbĘdne dla ich przezwyciĘżenia. Referat wygłoszony na III Plenum KC PZPR, 17.X.1959 (Warszawa: KIW) Gomułka, Władysław. 1969. Przemówienia 1968 (Warszawa : KIW). 
Khroutchtev et la culture. Texte intégral du discours du 8 mars 1963, avec notes et commentaires, non daté. (Paris : Revue Preuves)

Kim, Il Sung, Cuvres, 4, I - XII 1948. 1981. (Pyongyang : Editions en langues étrangères)

www.naenara.com.kp/fr/

http://www.cuba.cu/gobierno/discursos/

http://www.komunizm.eu/przemowienia_tekst.html

http://www.legrandsoir.info/Fidel-Castro-discours-du-26-juillet-1973.html

http://www.marxists.org/francais/lenin/oeuvres/vol_26.html

\section{NOTES}

1. Paul Marion (1899-1954), journaliste français, militant communiste devenu fondateur du PPF, puis membre du gouvernement de Vichy.

2. Hostis publicus, terme du droit romain. Le Sénat a utilisé cette expression pour désigner l'empereur Néron, avant son arrestation.

3. «Les chefs du parti Cadet, parti des ennemis du peuple, doivent être arrêtés et remis au tribunal révolutionnaire » (V. Lénine, CEuvres, t. 26, Paris-Moscou, p. 367).

(http://www.marxists.org/francais/lenin/oeuvres/vol_26.htm)

4. http://www.legrandsoir.info/Fidel-Castro-discours-du-26-juillet-1973.html

5. Voir à ce sujet notamment Alicja Kacprzak (2009).

6. Polska Partia Robotnicza (PPR)

7. Pravda, 24.05.1963, http://www.cvce.eu/content/publication/ 2004/2/10/7a223f24-7d74-434c-928b-85724626734d/publishable_fr.pdf

8. www.naenara.com.kp/fr/

9. http://www.komunizm.eu/przemowienia_tekst.html

10. Polska Zjednoczona Partia Robotnicza (PZPR)

11. Voir aussi Kamińska-Szmaj (2007).

12. www.cuba.cu/gobierno/discursos/

13. Elle se résume par la constatation que le pouvoir d'achat de la classe ouvrière est trop élevé, si bien qu'on achète trop de viande, ce qui entraîne sa pénurie sur les marchés.

14. http://www.cuba.cu/gobierno/discursos/

15. Khroutchtev et la culture. 1963. Texte intégral du discours du 8 mars 1963, avec notes et commentaires (Paris : Revue Preuves)

\section{RÉSUMÉS}

Selon Claude Lefort (1981), le totalitarisme se caractérise essentiellement par une double «clôture ", à savoir la destruction de l'espace public et l'abolition de la pluralité des opinions. Appréhendé selon ce point de vue, le discours totalitaire peut être compris comme un type de discours de domination qui, indépendamment de ses origines, tend à se superposer à tout dialogue social, en excluant des idéologies autres que celles qu'il prône lui-même. En accord avec 
l'hypothèse que certaines valeurs, plus que d'autres, appellent un certain type d'émotions, notre article se propose de montrer que le pathos négatif, avec son éventail d'instruments langagiers, peut être considéré comme un trait inhérent du discours totalitaire.

According to Claude Lefort (1981), totalitarianism is characterized by its double "closure," as it destroys the social space and abolishes plurality of opinions. In this view, totalitarian discourse can be seen as a kind of domination discourse that, independently of its origins, tends to superimpose the whole of the social dialogue, while excluding all ideas that are different from its own. In accordance with the hypothesis that certain values attract certain kind of emotions, this article attempts to demonstrate that negative pathos, together with the range of its linguistic instruments, can be considered an inherent feature of totalitarian discourse.

\section{INDEX}

Mots-clés : discours, émotions, pathos, totalitarisme

Keywords : discourse, emotions, pathos, totalitarianism

\section{AUTEUR}

\section{ALICJA KACPRZAK}

Université de Lodz 